\title{
Efficacy of Casein Phosphopeptide Amorphous Calcium Phosphate with and without Fluoride in Comparison with Fluoride Gel for Enamel Remineralization
}

\author{
Kaveh $\mathrm{K}^{1}$, Baharan Ranjbar 02*, Golnaz $\mathrm{A}^{3}$ and Vahid $\mathrm{D}^{4}$ \\ ${ }^{1}$ Department of Operative Dentistry, Tehran University of Medical Sciences, Iran \\ ${ }^{2}$ Department of Operative Dentistry, Qazvin University of medical sciences, Iran \\ ${ }^{3}$ Dentist, Tehran, Iran \\ ${ }^{4}$ Post graduate student of operative dentistry, Qazvin University of Medical Sciences, \\ Iran
}
Research Article
Volume 3 Issue 3
Received Date: July 16, 2018
Published Date: July 27, 2018
DOI: $10.23880 /$ oajds-16000187

*Corresponding author: Baharan Ranjbar Omidi, Assistant Professor, Department of operative dentistry, dental caries prevention research center, Qazvin University of medical sciences, Qazvin, Iran, Tel: +98 912 6447687; Email: baharanomidi@yahoo.com

\section{Abstract}

Background and Objectives: Remineralization, as a treatment modality, has attracted the attention of dental clinicians. Recent studies on the role of casein and calcium phosphate derived from milk showed that casein phosphopeptide (CPP) complex stabilizes the calcium phosphate and leads to remineralization of incipient carious lesions. This study aimed to assess and compare the efficacy of casein phosphopeptide amorphous calcium phosphate (CPP-ACP), casein phosphopeptide amorphous calcium phosphate fluoride (CPP-ACPF) and fluoride gel for remineralization of enamel carious lesions.

Materials and Methods: One-hundred extracted human premolars were divided into four groups of 25: artificial saliva (group A), CPP-ACP (group B), CPP-ACPF (group C) and fluoride gel (group D). The DIAGNOdent values of buccal tooth surfaces were measured at baseline, after demineralization and at seven, 14 and 21 days after remineralization.

Results: The mean DIAGNOdent value(remineralization rate)was 7.4, 6.96 and 8.16 in CPP-ACP, CPP-ACPF and fluoride gel groups, respectively at seven days, 7.04, 6.6 and 7 at 14 days and 6.48, 6.08 and 6.52, respectively at 21 days (ANOVA, $\mathrm{P}<0.05)$. At all three time points, remineralization of enamel lesions in the test groups was significantly higher than that in the control group but no significant difference was noted between the test groups in this respect. 
Conclusion: CPP-ACP, CPP-ACPF and fluoride gel all showed significant efficacy for remineralization of demineralized enamel lesions. Also, CPP-ACPF (GC MI Paste Plus) caused greater remineralization than CPP-ACP (GC Tooth Mousse) and fluoride gel due to the incorporation of $0.2 \% \mathrm{NaF}$ in its composition.

Keywords: Casein Phosphopeptide Amorphous Calcium Phosphate; Casein Phosphopeptide Amorphous Calcium Phosphate Fluoride; Fluoride Gel; DIAGNOdent; Remineralization

Abbreviations: CPP: Casein Phosphopeptide; ACPF: Amorphous Calcium Phosphate Fluoride; WSLs: White Spot Lesions; ACP: Amorphous Calcium Phosphate; QLF: Quantitative Light-induced Fluorescence; DIFOTI: Digital Imaging Fiber Optic Transillumination.

\section{Introduction}

Tooth decay is a chronic disease with slow progression, which is not often detectable at its onset. It starts with demineralization and release of calcium and phosphate ions leading to the formation of a subsurface lesion [1]. The pathological process of tooth destruction occurs following the activity of cariogenic microorganisms, which ferment carbohydrates and produce organic acids, causing demineralization of dental hard tissue [2]. Formation of white spot lesions (WSLs) is the first clinical symptom of caries, which can be considered as the first step of enamel demineralization. These lesions are detectable by the human eye and have a chalky white, opaque appearance on smooth tooth surfaces. They are more commonly found in the cervical third of the teeth $[3,4]$.

Treatment of WSLs often includes preventive measures and enhancement of remineralization. The logic behind the use of fluoride for caries prevention is based on its penetration into the crystalline network of minerals in dental hard tissue and subsequent formation of a resistant mineral phase against acid attacks and decreasing the enamel dissolution rate [5]. Fluoride ions stop the process of caries progression by forming fluorapatite or causing deposition of dissolved hydroxyapatite in the enamel structure [6]. Although topical application of fluoride can significantly prevent the development of WSLs [7,8], studies on the management of these lesions are limited [9].

In the recent years, calcium phosphate-based remineralization technologies have shown promising results as an adjunct to topical fluoride therapy for non- invasive treatment of incipient caries $[10,11]$. However, clinical studies in this respect are scarce [12]. Novel methods for enamel remineralization include the use of milk and milk products, which have shown promising results in caries prevention [3]. Recent studies on the role of casein and calcium phosphate derived from milk showed that casein phosphopeptide (CPP) complex stabilizes the calcium phosphate and leads to remineralization of incipient carious lesions [13].

The CPP molecules are comprised of phosphoseryls that significantly increase the solubility of calcium and phosphate by stabilizing the amorphous calcium phosphate (ACP) in alkaline and neutral conditions. CPP enhances the stabilization of calcium phosphate in aqueous environments with alkaline or neutral $\mathrm{pH}$ by 100 times. Calcium and phosphate ions released from the CPP are absorbed by the enamel prisms, reforming hydroxyapatite crystals [14]. Conventional radiography, quantitative light-induced fluorescence (QLF), microradiography, digital imaging fiber optic transillumination (DIFOTI) and DIAGNOdent are among the optical methods/tools uses for caries detection. DIAGNOdent is an instrument for non-invasive detection of incipient and non-activated carious lesions [15].

The mechanism of action of QLF is based on the inherent fluorescence of the enamel and its reduction following demineralization. However, some factors compromise the specificity of this method [16]. DIFOTI is the completed version of FOTI, which is based on the passage of light through an object [17]. It is a quantitative method for caries detection. In this method, a $0.5 \mathrm{~mm}$ thin fiber optic probe is used and it is an efficient tool for detection of proximal caries. However, enamel caries cannot be easily and accurately detected by this tool, and its main shortcoming is its low sensitivity $[16,18]$.

Considering the high prevalence of dental caries, this study aimed to assess the efficacy of CPP-ACP, CPP-ACPF and fluoride gel for enamel remineralization. 


\section{Open Access Journal of Dental Sciences}

\section{Materials and Methods}

This in vitro study was conducted on 100 sound permanent human premolars extracted for orthodontic treatment. All teeth were sectioned in mesiodistal direction into buccal and lingual halves using a diamond disc. The buccal half of the teeth was used for this study. All samples were mounted in dental stone and coded. A white paper measuring $4 \times 4 \mathrm{~mm}$ was placed on the teeth to confine the test area before applying the acid-resistant varnish.

DIAGNOdent (DIAGNODENT kavodentalpen 1290, Germany) was then used to measure the mineral content of the teeth. Samples with DIAGNOdent scores 0 to 7 (sound) were chosen for this study. Samples with a score larger than 7 (demineralized) were excluded. The teeth were then randomly divided into four groups of 25 . Each group was coded and the baseline DIAGNOdent value of each sample was measured. To artificially create carious lesions, each tooth was separately immersed in $20 \mathrm{~mL}$ of demineralizing solution for five hours and was then rinsed with ion-free water $\left(2.2 \mathrm{~m} \mathrm{MkH}_{2} \mathrm{PO}_{4}, 50 \mathrm{~m}\right.$ Macetic acid and $2.2 \mathrm{~m} \mathrm{MCaCl}_{2}$ ). The teeth were then tested with DIAGNOdent and samples showing score 8 or higher were chosen for further assessments.
Samples in each group were then immersed in remineralizing solution daily and were then stored in artificial saliva $\left(20 \mathrm{mmol} / \mathrm{l} \mathrm{NaHCO} 3,3 \mathrm{mmol} / \mathrm{l} \mathrm{NaH}_{2} \mathrm{PO}_{4}\right.$, $1 \mathrm{mmol} / \mathrm{l} \mathrm{CaCl}_{2}$ ) at room temperature and neutral $\mathrm{pH}$ for one week, two weeks and three weeks. Group A samples were rinsed with ion-free water and placed back in the plastic container containing artificial saliva. CPP-ACP was then applied on group B samples while CPP-ACPF was applied on group $C$ samples. Fluoride gel was applied on group D samples for four minutes and the samples were then rinsed with ion-free water. The samples were then immersed in artificial saliva. All samples were measured by DIAGNOdent at one, two and three weeks. Data were analyzed using SPSS version 20 (SPSS Inc., IL, USA) via ANOVA and t-test.

\section{Results}

A total of 100 premolar teeth were evaluated. No significant difference was found among the groups in DIAGNOdent scores before and after enamel demineralization. In other words, the groups were homogenous and matched in this respect $(\mathrm{P}>0.05)$. A shown in Table 1, significant differences were noted in DIAGNOdent scores at seven, 14 and 21 days after enamel remineralization $(\mathrm{P}<0.05$, Table 1$)$.

\begin{tabular}{|c|c|c|c|c|}
\hline Time & Group & Mean & Standard deviation & P value \\
\hline \multirow{4}{*}{$\begin{array}{l}\text { Immediately after } \\
\text { demineralization }\end{array}$} & Control & 8.88 & 0.88 & \multirow{16}{*}{0} \\
\hline & CPP-ACP & 9.48 & 1.8 & \\
\hline & CPP-ACPF & 9.68 & 1.86 & \\
\hline & Fluoride & 9.52 & 1.87 & \\
\hline \multirow{4}{*}{ At 7 days } & Control & 8.64 & 0.95 & \\
\hline & CPP-ACP & 7.4 & 1.4 & \\
\hline & CPP-ACPF & 6.96 & 1 & \\
\hline & Fluoride & 8.16 & 1.4 & \\
\hline \multirow{4}{*}{ At 14 days } & Control & 8.48 & 1 & \\
\hline & CPP-ACP & 7.04 & 0.88 & \\
\hline & CPP-ACPF & 6.6 & 1.1 & \\
\hline & Fluoride & 7 & 1.4 & \\
\hline \multirow{4}{*}{ At 21 days } & Control & 8.44 & 1 & \\
\hline & CPP-ACP & 6.48 & 0.9 & \\
\hline & CPP-ACPF & 6.08 & 1 & \\
\hline & Fluoride & 6.52 & 1.6 & \\
\hline
\end{tabular}

Table 1: DIAGNOdent scores in the four groups at different time points after enamel remineralization.

Figure 1 shows the DIAGNOdent scores of the groups at different time points after enamel remineralization. DIAGNOdent scores were significantly different at 7,14 and 21 days after enamel remineralization in the control group compared to CPP-ACP, CPP-ACPF and fluoride groups. 


\section{Open Access Journal of Dental Sciences}

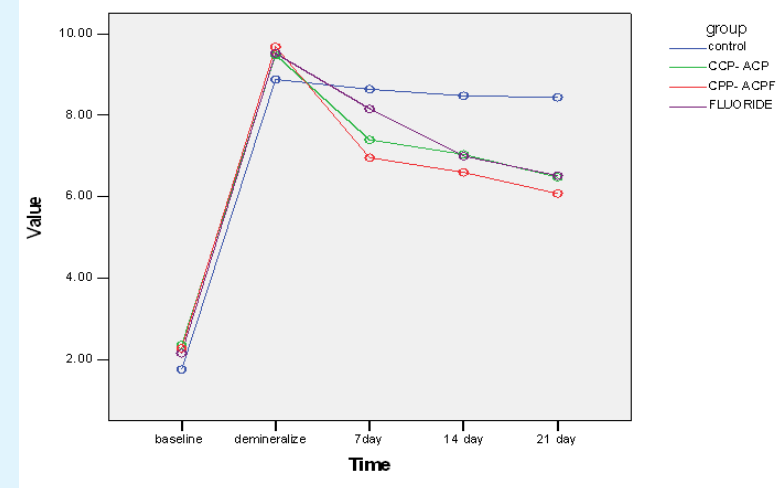

Figure 1: DIAGNOdent scores in the groups at different time points after enamel remineralization.

\section{Discussion}

Several studies have reported the optimal efficacy of topical fluoride therapy for enamel remineralization of incipient carious lesions and reduction of enamel solubility [19-22]. CPP-ACP is currently marketed under the brand name Tooth Mousse. It can stop the progression of caries since it decreases demineralization and enhances remineralization [23]. In the current study, enamel remineralization rate (DIAGNOdent score) was significantly different in the three groups of CPP-ACP, CPP-ACPF and fluoride at 7, 14 and 21 days. DIAGNOdent was used to assess the remineralization rate and the results showed that the mean remineralization rate (DIAGNOdent score) was the highest in CPP-ACPF group followed by the CPP-ACP and fluoride groups. Also, the remineralization rate in the control group was significantly different from that in all three test groups. However, the difference in this respect among the three test groups was not significant. These results were in line with those of Jayarajan, et al. [14] in 2011.

Taranath, et al. [24] in 2014 evaluated the role of CPPACP and its derivatives in remineralization of primary enamel lesions and reported results similar to ours despite the differences in methodology, sample size and testing environment.

Mehta, et al. in 2013 compared the efficacy of CPP-ACP and CPP-ACPF for enamel remineralization of teeth with artificially induced carious lesions using QLF [13]. They reported that both CPP-ACP and CPP-ACPF compared to artificial saliva caused enamel remineralization and were not significantly different. But, in contrast to our study, artificial saliva had a significant difference with CPP-ACP and CPP-ACPF at the end of day seven, which indicates that artificial saliva results in better remineralization than CPP-ACP and CPP-ACPF at the end of one week. This difference between our results and theirs may be due to the use of QLF in their study instead of DIAGNOdent.

In 2010, Lata, et al. [25] evaluated and compared the remineralization potential of fluoride and CPP-ACP for enamel lesions and showed that CPP-ACP was less effective than fluoride for remineralization of incipient enamel lesions. Also, they reported that combination of fluoride and CPP-ACP did not have a greater potential for enamel remineralization compared to fluoride alone.

\section{Conclusion}

Within the limitation of this study the following conclusions can be drawn:

a) CPP-ACP, CPP-ACPF and fluoride gel all showed significant efficacy for remineralization of demineralized enamel lesions.

b) CPP-ACPF (GC MI Paste Plus) caused greater remineralization than CPP-ACP (GC Tooth Mousse) and fluoride gel due to the incorporation of $0.2 \% \mathrm{NaF}$ in its composition.

c) At all three time points, remineralization of enamel lesions in the test groups was significantly higher than that in the control group (artificial saliva).

\section{References}

1. Al-Shaker SM, Nayif MM, Al-Sabawi NA (2014) Microhardness of Artificially Demineralized Enamel treated with Different Regimes of ACP-CPP and Fluoride Agents. International Journal of Enhanced Research in Science Technology \& Engineering 3(2): 102-107.

2. Derks A, Katsaros C, Frencken J, Van't Hof M, Kuijpers Jagtman A (2004) Caries-inhibiting effect of preventive measures during orthodontic treatment with fixed appliances. Caries Res 38(5): 413-420.

3. Chen F, Wang D (2010) Novel technologies for the prevention and treatment of dental caries: a patent survey. Expert Opin Ther Pat 20(5): 681-694.

4. Tostes M (2016) Remineralizing potential of CPP-ACP creams with and without fluoride in artificial enamel lesions: Authors' reply. Aust Dent J 61(3): 391.

5. ten Cate JM (1999) Current concepts on the theories of the mechanism of action of fluoride. Acta Odontol Scand 57(6): 325-329. 


\section{Open Access Journal of Dental Sciences}

6. de Leeuw NH (2004) Resisting the onset of hydroxyapatite dissolution through the incorporation of fluoride. The Journal of Physical Chemistry B 108(6): 1809-1811.

7. Benson PE, Parkin N, Millett DT, Dyer F, Vine S, et al. (2004) Fluorides for the prevention of white spots on teeth during fixed brace treatment. Cochrane Database Syst Rev (3): CD003809.

8. Stecksén Blicks C, Renfors G, Oscarson N, Bergstrand F, Twetman S (2007) Caries-preventive effectiveness of a fluoride varnish: a randomized controlled trial in adolescents with fixed orthodontic appliances. Caries Res 41(6): 455-459.

9. Bergstrand F, Twetman S (2003) Evidence for the efficacy of various methods of treating white-spot lesions after debonding of fixed orthodontic appliances. J Clin Orthod 37(1): 19-21.

10. Reynolds EC (2008) Calcium phosphate-based remineralization systems: scientific evidence? Aust Dent J 53(3): 268-273.

11. Reynolds EC, Cai F, Cochrane NJ, Shen P, Walker GD, et al. (2008) Fluoride and casein phosphopeptideamorphous calcium phosphate. J Dent Res 87(4): 344348.

12. Azarpazhooh A, Limeback H (2008) Clinical efficacy of casein derivatives: a systematic review of the literature. J Am Dent Assoc 139(7): 915-924.

13. Mehta R, Nandlal B, Prashanth S (2013) Comparative evaluation of remineralization potential of casein phosphopeptide-amorphous calcium phosphate and casein phosphopeptide-amorphous calcium phosphate fluoride on artificial enamel white spot lesion: an in vitro light fluorescence study. Indian J Dent Res 24(6): 681-689.

14. Jayarajan J, Janardhanam P, Jayakumar P (2011) Efficacy of CPP-ACP and CPP-ACPF on enamel remineralization-An in vitro study using scanning electron microscope and DIAGNOdent ${ }^{\circledR}$. Indian J Dent Res 22(1): 77-82.

15. Bahrololoomi Z, Musavi SA, Kabudan M (2013) In vitro evaluation of the efficacy of laser fluorescence (DIAGNOdent) to detect demineralization and remineralization of smooth enamel lesions. J Conserv Dent 16(4): 362-366.
16. Gomez J (2015) Detection and diagnosis of the early caries lesion. BMC oral health 15(1): S3.

17. Astvaldsdottir A, Ahlund K, Holbrook WP, de Verdier B, Tranaeus S (2012) Approximal Caries Detection by DIFOTI: In Vitro Comparison of Diagnostic Accuracy/Efficacy with Film and Digital Radiography. International journal of dentistry 2012: 326401.

18. Fejerskov 0, Kidd E (2009) Dental caries: the disease and its clinical management: John Wiley \& Sons.

19. Ambarkova V, Goršeta K, Glavina D, Škrinjarić I (2011) The effect of fluoridated dentifrice formulations on enamel remineralisation and microhardness after in vitro demineralization. Acta Stomatologica Croatica 45(3): 159-165.

20. Ambarkova V, Goršeta K, Jankolovska M, Glavina D, Škrinjarić I (2013) Effect of the fluoride gels and varnishes comparing to CPP-ACP complex on human enamel demineralization/ remineralization. Acta Stomatologica Croatica 47(2): 99-110.

21. Wu G, Liu X, Hou Y (2010) Analysis of the effect of CPP-ACP tooth mousse on enamel remineralization by circularly polarized images. Angle Orthod 80(5): 933-938.

22. Hicks MJ, Flaitz CM (1999) Enamel caries formation and lesion progression with a fluoride dentifrice and a calcium-phosphate containing fluoride dentifrice: a polarized light microscopic study. ASDC journal of dentistry for children 67(1): 21-28.

23. Ghafournia M, Tehrani HNA, Samimi P, Savabi 0 (2010) The effect of CPP-ACP on enamel attrition under erosive conditions. Journal of Isfahan Dental School 5(4): 224.

24. Taranath A, Pai D, Chakravarthy K (2014) The role of casein phosphopeptide-amorphous calcium phosphate products in remineralization of incipient enamel lesions and its substantivity. Journal of Experimental and Integrative Medicine 4(1): 67-70.

25. Lata S, Varghese N, Varughese JM (2010) Remineralization potential of fluoride and amorphous calcium phosphate-casein phospho peptide on enamel lesions: An in vitro comparative evaluation. J Conserv Dent 13(1): 42-46. 